\title{
the Investigation of the Language of Building Decoration Materials
}

\author{
Feng $\mathrm{He}$ \\ Art Design School of Guangxi Normal University , Guangxi China \\ 1057244610@qq.com
}

\begin{abstract}
Key words: Material language Emotion Decoration Materials
ABSTRACT: The relationship between design and decoration materials are inseparable. Different material languages can create different decorative styles. Different materials can create different spatial styles. Different materials carry diverse humanities, The relationship between design and decoration materials are inseparable. Different material languages can create different decorative styles. Different materials can create different spatial styles. Different materials carry diverse humanities.
\end{abstract}

\section{introduction}

The essence of the interaction between materials and people is that people understand the language of materials through non-verbal communication. In addition to the basic characteristics of the economic, physical and mechanical properties, the material has other special factors, such as the sense of touch, smell and hearing characteristics of the material. In a given environment, both conscious and unconscious communication between people and materials rely on the three properties of the material that act on the central nervous and sensory organs of the human being so that both the psychologically and psychologically affected. In the actual use of the material, these three properties of the material are generally one-piece and do not function in isolation.

As a linguistic tool, materials convey a particular meaning in a unique way of presentation, unique in nature, and in character. Understanding the material language helps us to use the material most effectively. Any combination of materials and their corresponding forms will derive their own unique linguistic form from the relationship between the material language and its form. A full understanding of these linguistic forms helps us understand the nature of the material language and make better use of the material.

\section{Material decorative language}

Material decorative performance is an important factor in the formation of decorative beauty, there are differences between the performance of different materials, the use of this difference can enrich the level of material decorative performance. The function of material decoration is to beautify the space by the decorative properties in its characteristics, which will improve the artistic effect of the main body. Its beautification effect is reflected by the color and texture of the material, and the use of different combinations of different materials will produce different decorative effects. The combination of white or light materials creates a comfortable, quiet, dark-colored combination of materials that enhances the stability and dignity of the space, in terms of the material's beauty of color. The use of materials on the light absorption, reflection of varying strength, highlighting or weakening the surface texture of the material, will have some space beautification function, enhance people's impression of space. 


\section{Emotional expression of material}

In addition to decorative materials can reflect the function, but also to express feelings. The material is rich in texture, rough, smooth, delicate, soft, hard, and its color can be summarized as cold, warm, gray, bright, dark and other light and shadow forms, which can reflect the designer's performance on the object of emotion. Traditional materials such as clay, tile, bone, and jade remind people of ancient things, giving them a rustic, pure, pure feeling. Apply such materials to the decoration, always bring classical emotional tendencies. In the use of modern materials, such as glass, reinforced concrete, fiber fabrics, and then extended to multi-media materials, the use of these media will have a modern flavor. Therefore, in order to convey a specific emotion, it is necessary to choose the right material.

Material texture can make people's psychological effects, causing Lenovo, and the association of the force is relatively strong. Like transparent, high-gloss materials, giving a pure, bright emotional tone, soil, sand and stone materials, usually make people feel the atmosphere of the country, metal material gives a cold, tough feeling; fiber products to people In a warm emotional tone. Therefore, the material selection, the realization of the carrier and attachment in the temperament is necessary. Starting from the artist's personal feelings, any material in life can become an element of creation, borrowing them to express individuality and incorporating a new creative method in modern decorative painting. The artists put gravel, metal particles, wood chips, glass, etc. Mixed into the paint to create, through the random or deliberate combination of materials to express their emotions and ideas. In the installation art, there is also the integration of industrial ready-mades with soil.

In interior design, designers' creations are often influenced and restricted by culture, incorporating cultural factors more or less into the design. It is impossible for any designer to design a space exactly as he wishes. They usually observe the surroundings by means of a certain style and mode, which reflects the spiritual metaphor of culture. A space that has been designed by integrating spiritual culture, the material point and spirituality used in the space give the material life and thought, so that the material has the spiritual connotation of the metaphor.

\section{Building decoration material language elements}

\section{Material language elements ---- texture}

In many perceptual experiences, the sense of touch is the experience of physical contact with the real thing. By tactilely experiencing the change of the material's roughness, smoothness or roughness, temperature and curvature, the instantaneous perception of the touch is agreed with the psychological experience. For the texture of the material, generally based on the human touch texture. Tactile pressure also known as pressure, the skin surface to withstand the pressure of objects or touch something produced when feeling; touch is not only an important means of human communication, but also can bring people psychological and physical pleasure and relaxed feeling, And assist people's visual identification work. Such as wood materials, metal materials, cloth materials and other decorative objects, are people can be directly touched the material, giving a smooth, soft, hard, cold and other different psychological feelings, people perceive this ability is a direct role in Brain, tactile feelings are non-logical thinking,

\section{Material language element ---Processing technology}

Based on the essential properties of the material, the material is processed and processed to give it the functions of decoration in addition to the most basic functions. Hard wood and steel are processed to create the decorative effect of the curve. Material characteristics of the process is 
mainly reflected in the means of its processing, such as steel smelting, filling, casting and other processing can be shaped to achieve the desired shape, and the logs through cutting, grinding and other processes will become Floor, wood and other decorative materials. The use of materials has a significant feature that is more rational and clever use of the characteristics of materials for modern processing, making the comprehensive function of the material be fully reflected.

\section{Material language element ---color}

Decorative effect is the first thing that catches the eye color, the basic style of decoration is determined by the color. Color can trigger a lot of human emotions. To metal materials, for example, according to its color can be divided into mainly black and non-ferrous metals. Warm colors in non-ferrous metals give gorgeous, rich feeling, such as gold and bronze, blue-gray bronze people feel very solemn, and more ancient copper used in the manufacture. These bring emotional experience to people vary, use the black soft steel or gold, soft steel gives solemn and sharp feeling, gold is gorgeous and soft. Material color plays an important role in man-made environment. Designers can create a colorful and full-fledged indoor environment as long as they master the habits of the users, their personalities and hobbies, and the creative, reasonable and matching material colors.

\section{Material language element ---Texture}

Texture refers to the body's own body shape and surface texture of the material, is the form factor of texture, reflecting the morphological characteristics of the material table, so that the texture of the material to reflect more specific and image. In interior design, the texture and texture of different decorative materials will give different psychological experience. Such as clear texture of wood, bamboo material gives a cordial, soft, warm feeling; with a false mark of false stone fierce, foul language; Reflective metal texture is not only hard and firm, strong tension, indifference, and beautiful and novel, Noble; glass gives a clean, bright and transparent feeling.

In the interior design, to show the texture properties of the material, it is necessary for the designer to compose the composition of the material texture, to reconcile the contrast of the texture of different materials, and to have different understanding of the atmosphere effect.

\section{Material language and the relationship between indoor space environment}

The purpose of indoor space environment decoration is to make people's work and living environment in space and visual effect to achieve the harmony between man and environment, and this harmony often depends largely on the choice of decorative materials, therefore, in progress Interior design should be fully familiar with the characteristics of a variety of decorative materials features, give full consideration to the language of a variety of decorative materials, according to the requirements of space environment, rational allocation and use of decorative materials.

Decorative materials itself has a unique attribute and aesthetic characteristics, contrast of different materials, set off, in the visual effects have different effects, there is both rival and harmonious rhythm beauty. In addition, the texture of the material in the human psychology reaction caused by association. Therefore, the choice of decorative materials, we must correctly grasp the character of the material, so that it is decorated with the spatial characteristics, decorative style, giving the material life. Interior design is usually through a variety of different combinations of materials, the use of contrast fusion decorative materials hard and soft texture, rough or exposed to meet the needs of different specifications of the indoor space environment, "harmonious" beauty. 


\section{Building decoration materials language form}

\section{Material language to adapt to the requirements of interior space function}

Designers should pay attention to the functional division of human activities when using materials. In such a limited space in the interior, designers combine materials into different forms to show the significance of the material in space. In an open space such as outdoor space, it has greater spatial continuity, based on space is made of material architecture, designers should fully demonstrate the structure of the material language in space. Material language plays a decisive role in the nature and size of the space. Materials make space real, not illusory. Even in the virtual world of human fantasies, those bizarre things, plots, images also originated in the real world by a variety of materials. The functions of each space are different and require different decorative materials to express the atmosphere in the room. For example, the living room is the center of activity of family members, and the atmosphere is delightful and joyous. The bedroom is a resting and sleeping room that requires quietness and privacy. Kitchen, bathroom need to be bright and clean, which is closely related to the color, texture and texture of selected materials.

\section{Material language to meet the requirements of indoor human style}

Decorative style and style in every nation, every era, every region is different, different regions in the concept of decoration, customs are different, so the choice and use of materials are not the same. Decorative style, style, the requirements are subject to a greater degree of material constraints. Therefore, the formation and development of decorative styles and styles are often closely related to the selection, application and processing of materials as well as the existing conditions of the local decorative materials at that time.

For example, wood as the main decoration material, its texture warm and moist, beautiful texture, good coloring, has always been the first choice for indoor timber. Due to the differences in culture and aesthetics, wood is often used as an indispensable material in decorative systems in many oriental countries. It has a deeper understanding of the texture of wood and creates a color picture with one of the main features of structure Frame decorative system, highlighting the oriental unique decorative style art form. Stone texture hard, natural texture and color changeable, conclusive and good characteristics, as the most ideal structure of the building materials, but also the interior interface paving the high-grade timber; many Western countries and regions to use stone to create a column Style arch represented by the sculpture of a strong interface decoration system, as well as the popular European-style interior design of the most representative of the performance of the elements. The metallic material is cold and smooth texture, simple color, plastic molding processing strong, but requires the support of the level of modern processing technology, so a large number of metal materials as the interior decoration materials represent the most typical modern decorative style.

\section{Conclusion}

Various materials have their own characteristics, we study material language should be the choice of materials and indoor environment function, the style to be closely linked to the use and composition of materials to meet the architectural and interior functional character, in line with the overall construction Art style. Material selection should be in the premise of sustainable development strategy, adhere to the principle of people-oriented rational selection, design suitable for people's aesthetic needs, comfortable building and indoor space environment. 


\section{Acknowledgements}

This work was funded by the Guangxi Municipal Commission of Education Plan (KY2015ZD022).

\section{References}

[1] Cong Zhou. Interior decoration form composition (2008), China Building Industry Press 